\title{
Platelet Rich Plasma Treatment of Androgenetic Alopecia in Men and Women
}

\section{Introduction}

Androgenic Alopecia (AGA) is one of the most common hair loss complaints in men and women. The population frequency of AGA varies with ethnicity but as a rough generalization up to $70 \%$ of men and $40 \%$ of women will experience some degree of AGA in their life time. In most men AGA develops with a distinctive "patterned" hair line recession. In women the presentation may be less clear typically women will develop a diffuse thinning over the top of the scalp yielding a "Christmas tree" pattern with more thinning towards the front though the frontal hairline is maintained [1]

The long-term treatment of AGA with the FDA approved topical minoxidil and oral finasteride therapy reported several side effects including scalp irritation facial hypertrichosis and loss of libido. So the use of a newer modality of Platelet-Rich Plasma (PRP) has attracted attention in the treatment of AGA because of its beneficial effect with minimal or even no side effects $[2,3]$.

Platelet-Rich Plasma (PRP) can be defined as an autologous concentration of human platelets in a small volume of plasma. This concentrate contains various Growth Factors (GFs) that stimulate cell proliferation and differentiation including PDGF, fibroblast FGF, HGF, TGF and VEGF [4]. These growth factors are released once the platelets therein are activated either by calcium chloride, thrombin or fibrinogen. All seem equally effective in activating the platelets ex vivo [5].

Activated autologous PRP has been reported to induce the proliferation of dermal papilla cells by up-regulating Fibroblast Growth Factor 7 (FGF-7) and $\beta$-catenin as well as Extracellular Signal-related Kinase (ERK) and Akt signaling. Anagen associated angiogenesis has been suggested as one of the important factors in active hair growth. In addition the angiogenic factor of Vascular Endothelial Growth Factor (VEGF) also originated from the keratinocytes in the outer root sheath and fibroblasts in dermal papilla [6]. Therefore injection of PRP has been demonstrated to improve cutaneous ischemic conditions and to increase vascular structures around hair follicles [7].

The use of PRP has attracted attention in many fields of medicine as in bone grafts, teeth osteosynthesis, wound healing, tendinopathy, ligament sprain, muscle strain, osteoarthrosis, fracture non union and face rejuvenation [8].

In 2006 Uebel and colleagues reported the application of PRP for male pattern baldness surgery. Implanting follicular units with PRP raised the hair yield rate probably because of the partial effects of GFs in PRP [9].

The aim of this study was to assess the efficacy and safety of autologous platelet-rich plasma injection for treatment of AGA.

\section{Journal of}

Clinical \& Investigative Dermatology

\section{Mohamed A Toama ${ }^{1^{*}}$, Elsayed M Khater ${ }^{1}$ and Mohamed Soliman ${ }^{1}$}

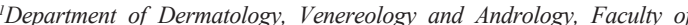
Medicine, Zagazig Univeristy, Egypt

\section{*Address for Correspondence}

Mohamed A Toama, Department of dermatology, venereology and andrology, Faculty Of Medicine, Zagazig Univeristy, Egypt, Tel: 01143156920; E-mail: abdesawy@hotmail.com

Submission: 07 July, 2017

Accepted: 25 July, 2017

Published: 01 August, 2017

Copyright: () 2017 Toama MA, et al. This is an open access article distributed under the Creative Commons Attribution License, which permits unrestricted use, distribution, and reproduction in any medium, provided the original work is properly cited

\section{Patients and Methods}

\section{Patients}

This study was carried out in the Department of Dermatology and Venereology, Faculty of Medicine, Zagazig University Hospitals in the period from May 2014 till April 2015. Forty males and females patients of different age, duration and grades of AGA were included in our study. The diagnosis of AGA was clinically established by the characteristic distribution of frontal and vertex hair in males and the chrismus tree pattern of diffuse hair loss at middle hairline in females. Also it was dermoscopically established by the characteristic hair diameter diversity and peripilar brown depressions (peripilar signs). Cases included in this study had Norwood-Hamilton Scale types I to V for men and Ludwig Scale types I and $\Pi$ for women. The study had the approval of The Institutional Review Board (IRB) at Zagazig University and all patients signed an informed consent before inclusion into the study.

Exclusion criteria included platelet dysfunction syndrome, critical thrombocytopenia, sepsis, acute and chronic infections, chronic liver disease, uncontrolled diabetes mellitus. Anticoagulant therapy or aspirin should be stopped at least 3 days before the procedure. Systemic use of corticosteroids or corticosteroid injection at the scalp should be stopped within one month before the study. Past history of keloidal scarring and patients with severe systemic illness or malignancy were also excluded.

All patients were subjected to:

- Full history taking: Personal history, present history, history of drug intake and family history of AGA.

- General examination.

- Dermatological examination of hair density to assess the AGA grade, scalp skin, eye brows, eye lashes, facial and body hair, signs of acne.

- Investigations including complete blood picture, prothrombin time and partial thromboplastin time. 
Citation: Toama MA, Khater EM, Soliman MI. Platelet Rich Plasma Treatment of Androgenetic Alopecia in Men and Women. J Clin Investigat Dermatol. 2017;5(2): 5

ISSN: 2373-1044

Participants were randomly divided into two groups: Group A: Included twenty patients (11 males and 9 females) aged 18- 45 years. They were treated with five sessions of injections with platelet-rich plasma (one session every two weeks).

Group B: Included twenty patients ( 8 males and 12 females) aged 19 - 40 years. They were treated with five sessions of injections with saline (one session every two weeks).

\section{Methods}

\section{Preparation of PRP}

PRP was prepared using a double spin method. The patient was first sent to a clinical pathology laboratory where $15 \mathrm{ml}$ of venous blood was aspirated from the patient by venipuncture of the median cubital forearm vein using a 21-gauge butterfly needle.

The blood was collected in five special $3 \mathrm{ml}$ sterile vacutainer tubes containing an anticoagulant $\mathrm{Na}$ Citrate $3.8 \%$. The citrated blood was centrifuged at $1,700 \mathrm{rpm}$ for 15 minutes at room temperature separates red blood cells from plasma which contains "buffy coat" (white blood cells and platelets).

The plasma was gently aspirated from each test tube into a syringe and transferred to another tube then centrifuged again at 3,000 rpm for 10 minutes at room temperature thus obtaining a two-part plasma. The upper part consisting of Platelet Poor Plasma (PPP) and the lower part consisting of Platelet-Rich Plasma (PRP). The PPP was first gently aspirated to avoid its mixing up with the PRP. The residual PRP was subsequently aspirated from each test tube and prepared for activation by calcium chloride in the proportion of $0.1 \mathrm{ml}$ of $\mathrm{CaCl}_{2}$ per $0.9 \mathrm{ml}$ of PRP thus obtaining a concentration of activated PRP $[4,7]$. Approximately $3 \mathrm{ml}$ of PRP was produced this PRP was then injected intradermally through a 30 gauge needle.

\section{Technique of injection}

The injection applied intradermally using $1 \mathrm{ml}$ syringe and 30 guage needle. Anatomical injection sites on the scalp frontal, parietal and vertex areas. Prior to injection the target scalp surface was cleaned thoroughly with alcohol pads. Patients received five injections at weeks $0,2,4,6,8$ and were observed for 12 weeks and 6 months. Less than $0.1 \mathrm{ml}$ is injected per site and approximately $1 \mathrm{~cm}$ between injection sites in the selected areas of scalp. When the injection was finished it was necessary to compress the points which bleed within a few seconds. Gentle massaging of scalp was done after the procedure.

\section{Evaluation of the results}

The results were evaluated by digital camera and dermoscopy (Digital Microscopy) by comparing the pre and post-improvement photographs taken at $1^{\text {st }}$ session (base line) 3 and 6 months from the $1^{\text {st }}$ session. The distances from nasal tip and upper part of the auricular base to the dermoscopically photographed sites were measured and the same locations were thus able to be selected accurately whenever needed.

The number of all hairs in a $1.0 \times 1.0 \mathrm{~cm}$ area was counted in dermoscopic images. Mean cross sections of all hairs in the

$1 \mathrm{~cm}^{2}$ area were calculated using the measured data by

$$
A=\pi r^{2}(\pi=3.14)
$$

\section{Side effects}

Pain during the session was assessed by the participants and graded as mild, moderate and severe. Any side effects observed such as erythema, burning sensation, swelling, bruising, headache at each session were recorded.

\section{Statistical Analysis}

All data were analyzed using SPSS 15.0 for windows (SPSS Inc, Chicago, IL, USA). Continuous data are expressed as the mean \pm SD \& median (range) and the categorical data are expressed as a number (percentage). Student t-test, ANOVA (F), Chi-Square $(\chi 2)$ and Spearman's rank correlation coefficient were used when appropriate.

\section{Results}

This study was conducted on 40 patients with AGA. The 40 patients were divided randomly into 2 groups (A and B).

\section{Group (A) platelet rich plasma injection}

This group included twenty patients in that 11 males (55\%) and 9 females (45\%). Their ages ranged from 18 to 45 years old with a mean of $28.45 \pm 6.58$.

\section{Group (B) Saline injection}

This group included twenty patients, 8 males (40\%) and 12 females $(60 \%)$. Their ages ranged from 19 to 40 years old with a mean of $28.8 \pm 7.58$. The demographic data of the two groups are shown in (Table 1).

\section{Therapeutic Response}

The response to treatment was assessed using digital camera and by dermoscopy (digital microscope)

\section{Hair count}

Mean numbers of hairs were $37.35 \pm 7.49,44.2 \pm 5.87$ at the experimental site $\left(1 \mathrm{~cm}^{2}\right)$ before administration of PRP and saline in groups A and B respectively. After 3 months when patients had received all five treatments mean numbers of hairs were $45.4 \pm 9.25$, $44.95 \pm 10.42 / \mathrm{cm} 2$ respectively. After 6 months mean numbers of hairs were $56.65 \pm 10.99,46.55 \pm 10.27$. Rates of increase at 3 months were $21.5 \%, 1 \%$ in groups A and B. At 6 months, rates of increase were $24.7 \%$ and $3.5 \%$ respectively. These results showed that group A exhibited greater mean number of hairs than the control group (group B) (Table 2).

\section{Hair cross section}

Mean cross-sections of hairs were $5.24 \pm 1.63$ and $7.22 \pm 2.43$ $\left(\mathrm{x} 10^{-4} \mathrm{~mm}\right)$ in the experimental site $\left(1 \mathrm{~cm}^{2}\right)$ before administration of

Table 1: Demographic data of the two studied groups.

\begin{tabular}{|c|c|c|c|c|c|c|}
\hline & \multicolumn{2}{|c|}{ Group A } & \multicolumn{2}{|c|}{ Group B } & \multirow[b]{2}{*}{$F$} & \multirow[b]{2}{*}{$\mathbf{P}$} \\
\hline Age(years) & & & & & & \\
\hline $\mathrm{X} \pm \mathrm{SD}$ & \multicolumn{2}{|c|}{$28.45 \pm 6.58$} & \multicolumn{2}{|c|}{$28.8 \pm 7.58$} & \multirow{2}{*}{-.156} & \multirow{2}{*}{0.877 (NS) } \\
\hline Range & \multicolumn{2}{|c|}{$18-45$} & \multicolumn{2}{|c|}{$19-40$} & & \\
\hline Gender & $\mathbf{N}$ & $\%$ & $\mathbf{N}$ & $\%$ & $x^{2}$ & $\mathbf{P}$ \\
\hline Male & 11 & 55 & 8 & 40 & \multirow{2}{*}{0.9} & \multirow{2}{*}{0.342 (NS) } \\
\hline Female & 9 & 45 & 12 & 60 & & \\
\hline
\end{tabular}


Citation: Toama MA, Khater EM, Soliman MI. Platelet Rich Plasma Treatment of Androgenetic Alopecia in Men and Women. J Clin Investigat Dermatol. 2017;5(2): 5

ISSN: 2373-1044

PRP and saline in groups A \& B respectively. At 3 months they were $7.35 \pm 2.18$ and $7.34 \pm 2.16\left(\times 10^{-4} \mathrm{~mm}^{2}\right)$. At 6 months they were 10.97 \pm 2.98 and $7.34 \pm 2.26$. Rates of increase at 3 months were $40 \%$ and $1.5 \%$ respectively. At 6 months rates of increase were $49 \%$ and $1 \%$ respectively. Significant differences were seen after 6 months between group A and group B (Tables 3,4).

\section{Regarding photographic assessment}

Group A: Showed excellent improvement in 2 patients (10\%), very good improvement in 8 patients (40\%), good improvement in 7 patients (35\%) and mild improvement was detected in 3 patients (15\%).

Group B: Showed no improvement in 18 patients (90\%) and mild improvement in 2 patients (10\%) (Table 5).

\section{Side effects}

The pain was noticed only during the procedure. It was quite tolerable with no need for analgesic after treatment or discontinuation of the sessions. No difference in the pain severity was noticed between male and female patients. No serious or persistent side effects were

Table 2: Hair count before 3 months and after 6 months PRP (group A) \& saline (group B) administration

\begin{tabular}{|c|c|c|c|c|}
\hline Hair count $\left(1 \mathrm{~cm}^{2}\right)$ & Group A & Group B & $\mathbf{t}$ & $\mathbf{P}$ \\
\hline \multicolumn{5}{|l|}{ Base } \\
\hline$X \pm S D$ & $37.35 \pm 7.49$ & $44.2 \pm 5.87$ & \multirow{2}{*}{-3.22} & \multirow{2}{*}{$0.002^{*}$} \\
\hline Range & $25-51$ & $37-52$ & & \\
\hline \multicolumn{5}{|l|}{ After 3 months } \\
\hline$X \pm S D$ & $45.4 \pm 9.25$ & $44.95 \pm 10.42$ & \multirow{2}{*}{0.144} & \multirow{2}{*}{0.886} \\
\hline Range & $27-57$ & $35-49$ & & \\
\hline \multicolumn{5}{|l|}{ After 6 months } \\
\hline$X \pm S D$ & $56.65 \pm 10.99$ & $46.55 \pm 10.27$ & \multirow{2}{*}{3.003} & \multirow{2}{*}{$0.005^{*}$} \\
\hline Range & $34-67$ & $30-63$ & & \\
\hline
\end{tabular}

Table 3: Hair cross sections before 3 months and 6 months after PRP (group A) $\&$ saline (group B) administration.

\begin{tabular}{|c|c|c|c|c|}
\hline Hair cross section $\left(\times 10^{-4} \mathrm{~mm}\right)$ & Group A & Group B & $\mathbf{T}$ & $\mathbf{P}$ \\
\hline \multicolumn{5}{|l|}{ Base } \\
\hline $\mathrm{X} \pm \mathrm{SD}$ & $5.24 \pm 1.63$ & $7.22 \pm 2.43$ & \multirow{2}{*}{-2.576} & \multirow{2}{*}{0.074} \\
\hline Range & $3.4-10.1$ & 2.9-8.2 & & \\
\hline \multicolumn{5}{|l|}{ After 3 months } \\
\hline $\mathrm{X} \pm \mathrm{SD}$ & $7.35 \pm 2.18$ & $7.33 \pm 2.16$ & \multirow{2}{*}{0.029} & \multirow{2}{*}{0.977} \\
\hline Range & $4.1-12.1$ & $4.5-12$ & & \\
\hline \multicolumn{5}{|l|}{ After 6 months } \\
\hline $\mathrm{X} \pm \mathrm{SD}$ & $10.97 \pm 2.98$ & $7.34 \pm 2.26$ & \multirow{2}{*}{2.823} & \multirow{2}{*}{$<0.001^{* *}$} \\
\hline Range & $4.2-14$ & $5.3-12.2$ & & \\
\hline
\end{tabular}

Table 4: Hair count and hair cross section before and 6months after PRP (group A) \& saline (group B) administration.

\begin{tabular}{|c|c|c|c|c|}
\hline & Experimental area $\left(1 \mathrm{~cm}^{2}\right)$ & $\mathrm{X} \pm \mathrm{SD}$ & T test & $\mathbf{P}$ \\
\hline \multirow{4}{*}{ Group A } & Hair count base & $37.35 \pm 7.49$ & \multirow{2}{*}{-10.7} & \multirow{2}{*}{$<0.001^{* *}$} \\
\hline & Hair count after 6 months & $56.65 \pm 10.99$ & & \\
\hline & Hair cross base & $5.24 \pm 1.63$ & \multirow{2}{*}{-13.4} & \multirow{2}{*}{$<0.001^{* *}$} \\
\hline & Hair cross after 6 months & $10.97 \pm 2.98$ & & \\
\hline \multirow{4}{*}{ Group B } & Hair count base & $44.2 \pm 5.87$ & \multirow{2}{*}{-1.7} & \multirow{2}{*}{0.09} \\
\hline & Hair count after 6 months & $46.55 \pm 10.27$ & & \\
\hline & Hair cross base & $7.22 \pm 3.6$ & \multirow{2}{*}{-1.9} & \multirow{2}{*}{0.06} \\
\hline & Hair cross after 6 months & $7.34 \pm 2.26$ & & \\
\hline
\end{tabular}

detected in both groups. The differences between the two groups in the degree of pain $(P=0.5)$ and the side effects $(P=0.7)$ were statistically non significant (Tables 6,7).

\section{Case presentation (Figures 1,2).}

\section{Discussion}

Androgenetic Alopecia (AGA) a hereditary and androgen dependent progressive thinning of the scalp hair in a defined pattern is a common dermatological disorder affecting men and women with significant negative impact on their social and psychological well being with loss of self-esteem, depression, neuroticism, feeling unattractive and introversion [2].

Table 5: Photographic assessment of the two studied groups.

\begin{tabular}{|c|c|c|c|c|c|c|}
\hline \multirow{2}{*}{} & \multicolumn{2}{|c|}{ Group A } & \multicolumn{2}{c|}{ Group B } & $\mathbf{X}^{2}$ & \multicolumn{1}{c|}{$\boldsymbol{P}$} \\
\cline { 1 - 5 } & $\mathbf{N}$ & $\mathbf{( \% )}$ & $\mathbf{N}$ & $\mathbf{( \% )}$ & 35.2 & $<0.001^{* *}$ \\
\hline No improvement & 0 & $(0 \%)$ & 18 & $(90 \%)$ & & \\
\hline Mild improvement & 3 & $(15 \%)$ & 2 & $(10 \%)$ & & \\
\hline Good improvement & 7 & $(35 \%)$ & 0 & $(0 \%)$ & & \\
\cline { 1 - 4 } $\begin{array}{c}\text { Very Good } \\
\text { improvement }\end{array}$ & 8 & $(40 \%)$ & 0 & $(0 \%)$ & & \\
\cline { 2 - 4 } $\begin{array}{c}\text { Excellent } \\
\text { improvement }\end{array}$ & 2 & $(10 \%)$ & 0 & $(0 \%)$ & & \\
\hline
\end{tabular}

Table 6: Pain in the two studied groups.

\begin{tabular}{|c|c|c|c|c|}
\hline & Group A & Group B & X & P \\
\hline Mild & $13(65 \%)$ & $12(60 \%)$ & & \\
\cline { 1 - 3 } Moderate & $6(30 \%)$ & $5(25 \%)$ & \multirow{2}{*}{1.01} & \multirow{2}{*}{0.5} \\
\hline Severe & $1(5 \%)$ & $3(15 \%)$ & & \\
\hline
\end{tabular}

Table 7: Side effects in the two studied groups.

\begin{tabular}{|c|c|c|c|c|}
\hline & Group A & Group B & X & P \\
\hline NO & $10(50 \%)$ & $9(45 \%)$ & \multirow{2}{*}{1.9} & 0.7 \\
\cline { 1 - 3 } Erythema & $2(10 \%)$ & $3(15 \%)$ & & \\
\hline Burning & $0(0 \%)$ & $1(5 \%)$ & & \\
\hline Bruising & $1(5 \%)$ & $2(10 \%)$ & & \\
\hline Headache & $7(35 \%)$ & $5(25 \%)$ & & \\
\hline
\end{tabular}

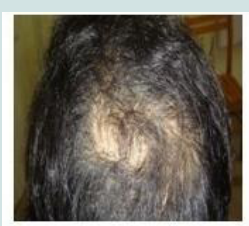

a

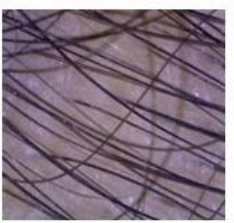

d

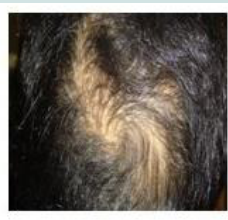

b

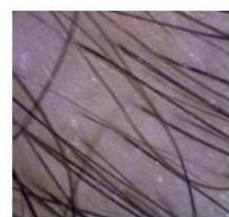

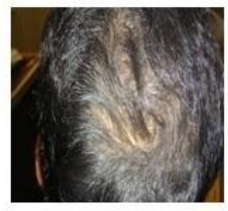

C

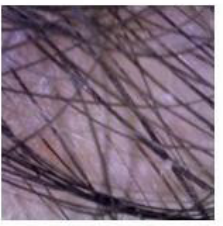

f
Figure 1: Male patient grade I AGA in group A showed very good improvement $(a-c)$ digital images $(d-f)$ dermoscopic images $(a, d)$ before $(b, e) 3$ months after and $(\mathrm{c}, \mathrm{f}) 6$ months after PRP injection. 


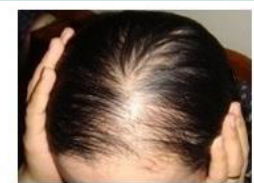

a

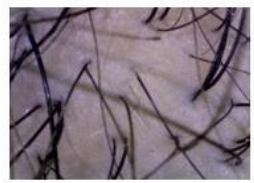

d

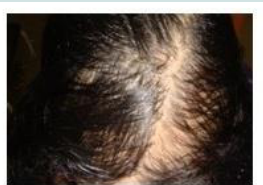

b

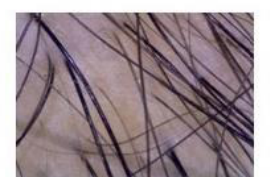

e

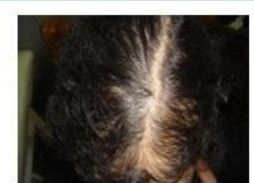

C

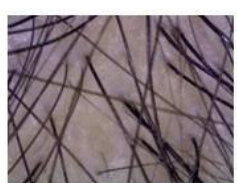

f
Figure 2: Female patient grade II AGA in group A showed very good improvement (a-c) digital images (d-f) dermoscopic images $(a, d)$ before $(b, e)$ 3 months after and (c,f) 6 months after PRP injection.

Treatment options of AGA to date include finasteride or minoxidil scalp surgery involving excision of bald scalp, scalp flaps, as well as transplantation. Also red lasers claim to stimulate hair growth through "photo-biostimulation" of the hair follicles. Finasteride and minoxidil are the only drugs that FDA has approved for the treatment of male pattern baldness alone or in combination. However there are several reported side effects including loss of libido and increase in other body hairs. The use of a newer modality of Platelet-Rich Plasma (PRP) has shown beneficial effect. PRP has been used in the past to prevent infection and speed up the wound healing process by reducing bleeding and swelling after surgery by the plastic, dental, general, neuro and orthopedic surgeons [10].

This study was conducted to evaluate the efficacy and safety of intradermal injection of PRP in treatment of androgenetic alopecia. The study included 40 patients divided into two groups $(A, B)$ and each included 20 patients complaining of AGA. Group A has been treated by intradermal injection of platelet rich plasma, while group $B$ has been treated with intradermal injection of saline.

The present study revealed promising results about using platelet rich plasma injection. The hair of AGA patients has improved after treatment with platelet rich plasma in all the patients of group A. Mean numbers and mean cross-sections of hairs were $37.35 \pm 7.49$ and5.24 $\pm 1.63\left(\mathrm{x} 10^{-4} \mathrm{~mm}\right)$ at the experimental site $\left(1 \mathrm{~cm}^{2}\right)$ before administration of PRP. After 3 months when patients had received all five treatments they were $45.4 \pm 9.25$ and $7.35 \pm 2.18\left(\times 10^{-4} \mathrm{~mm} /\right.$ $\mathrm{cm}^{2}$ ). After 6 months mean numbers of hairs were $56.65 \pm 10.99$ and $10.97 \pm 2.98$. Rates of increase at 3 months were $21.5 \%$ and $40 \%$. At 6 months rates of increase were $24.7 \%$ and $49 \%$.

These results were in agreement with the results reported by Takikawa, et al. [4]. Participants were 26 volunteers with thin hair who received five local treatments of $3 \mathrm{ml}$ of Dalteparin / Protamine Metaloprotienase (PRP\&D/P MPs) (13 participants) or PRP and saline (control, 13 participants) at 2-3 weeks intervals and were evaluated for 12 weeks. Significant differences were seen in hair cross section more than hair numbers in PRP and PRP\&D/P MP injections.

Greco, et al. and Lopez, et al. observed a significant increase in hair diameter and hair density respectively with the minimally invasive technique in which the scalp is punctured with microneedle roller of
$1 \mathrm{~mm}$ fine needles followed by interfollicular injections of PRP (or by using mesogun) over the treated area $[11,12]$.

Kang, et al. injected the scalps of 13 patients with CD34+ cell containing PRP preparation and 13 patients were treated with interfollicular placental extract injection as a control. Results 3 months after the first treatment the patients presented clinical improvement in the mean number of hairs $(\mathrm{P}<0.0001)$ and mean hair thickness $(\mathrm{P}<0.0001)$ compared with baseline. The PRP treatment presented a higher degree of improvement than placental extract treatment in hair thickness $(\mathrm{P}=0.027)$ and overall clinical improvement $(\mathrm{P}=$ 0.023) [13].

Cervelli, et al. showed a significant increase in the means of hair count (for the treatment area) and hair density (number of hairs/ $\mathrm{cm} 2$ ) after three months with a mean increase of 18.0 and 27.7 compared to baseline (control versus treatment $\mathrm{P}<0.0001$ ) [13]. In addition terminal hair density improved significantly by $27.0 \pm 15.3$ in the treatment area compared to baseline.

These results were not in agreement with Park, et al. who tried PRP injection on half of the scalp of one patient with AGA and the other side with saline as a control for 4 sessions (1 week interval) [14]. They observed significant increase in growth rate and hair density, but no change in hair thickness. This disagreement may attribute that this study used only one patient.

Uebel, et al. observed a significant difference in the yield of follicular units when comparing the experimental with the control areas of the scalp. The areas treated with platelet plasma growth factors demonstrated a yield of 18.7 follicular units per $\mathrm{cm}^{2}$, while the control areas yielded 16.4 follicular units per $\mathrm{cm}^{2}$, an increase in follicular density of $15.1 \%$ [9].

Greco and Brandt utilized PRP in all phases of a hair transplant procedure and infused PRP into scalps of patients prior to hair transplantation. These patients seemed to have thicker looking hair when compared to patients who had not had PRP therapy [15].

Sorbellini, et al. carried out an in vitro study on 50 patients. Twelve follicles were taken from each patient, of which four follicles were placed in PRP, four in Ringer's solution, and four in a standard solution. The authors then measured mitotic activity. The results showed a significant increase of mitotic activity and a reduction in the apoptotic process in the PRP group [16].

Cervelli, et al. and Takikawa, et al. examined head skin histologically [3,4]. Biopsies were performed from consenting participants before first injection and after 3 months of PRP treatment using a $4 \mathrm{~mm}$ disposable biopsy punches in perpendicular pattern and stained with H\&E reagent. After administration of PRP\&D/P MPs and PRP microscopic findings showed thickened epithelium, proliferation of collagen fibers and fibroblasts and greater numbers of blood vessels around hair follicles.

Antiapoptotic effects of activated PRP have been suggested as one of the major contributing factors stimulating hair growth. PRP induced activation of antiapoptotic regulators, such as the Bcl-2 protein and Akt signalling, prolongs the survival of dermal papilla cells during the hair cycle. In addition, the upregulation of FGF-7 and b-catenin signaling pathways with PRP treatment is suggested to 
Citation: Toama MA, Khater EM, Soliman MI. Platelet Rich Plasma Treatment of Androgenetic Alopecia in Men and Women. J Clin Investigat Dermatol. 2017;5(2): 5

stimulate hair growth by inducing follicular stem cell differentiation as well as prolonging the anagen phase of the hair growth cycle [7].

In the present work, No serious or persistent side effects were detected. Park, et al. and Takikawa, et al. reported that the patients experienced only temporary pain and swelling at the injection sites and these symptoms disappeared with in a day but no side effects with either injection such as hematoma or infection $[4,14]$.

In Kang, et al. study side-effects of treatment included pain during the treatment, transient post-treatment erythema and edema in the PRP treatment. Other possible side-effects such as secondary bacterial or viral infection, post-therapy blister formation, hypopigmentation, worsening of hair loss or scarring were not observed [13].

\section{Conclusion}

Treatment of AGA with Platelet-Rich Plasma (PRP) is a promising easy-to-use technique, performing favorably more than other traditional methods of AGA treatment. Good results were observed in increasing hair density and the emotional status of the patients improved. No serious or persistent side effects were detected. Although the method needs further validations initial results are encouraging and promising, particularly for those cases in which reparative processes are required.

\section{References}

1. Sinclair R, Patel M, Dawson TL, Yazdabadi A, Yip L, et al. (2011) Hair loss in women: Medical and cosmetic approaches to increase scalp hair fullness. $\mathrm{Br}$ J Dermatol 165: 12-18.

2. Betsi E, Germain E, Kalbermatten DF, Tremp M, Emmeneggeret V (2013) Platelet-rich plasma injection is effective and safe for the treatment of alopecia. Eur J Plast Surg 36: 407-412.

3. Cervelli V, Garcovich S, Bielli A, Cervelli G, Curcio BC, et al. (2014) The effect of autologous activated platelet rich plasma (AA-PRP) injection on pattern hair loss: Clinical and histomorphometric evaluation. Biomed Res Int 2014: 760709 .
4. Takikawa M, Nakamura S, Nakamura S, Ishirara M, Kishimoto S, et al. (2011) Enhanced effect of platelet-rich plasma containing a new carrier on hair growth. Dermatol Surg 37: 1721-1729.

5. Marx RE (2004) Platelet-rich plasma: Evidence to support its use. J Ora Maxillofac Surg 62: 489-496.

6. Semalty M, Semalty A, Joshi GP, Rawat MS (2011) Hair growth and rejuvenation: An overview. J Dermatolog Treat 22: 123-132

7. Li Z, Choi HI, Choi DK, Sohn KC, Im M, et al. (2012) Autologous platelet-rich plasma: A potential therapeutic tool for promoting hair growth. Dermatol Surg 38: $1040-1046$

8. Eppley BL, Pietrzak WS, Blanton M (2006) Platelet-rich plasma: A review of biology and applications in plastic surgery. Plast Reconstr Surg 118: 147159.

9. Uebel CO, da Silva JB, Cantarelli D, Martins P (2006) The role of platelet plasma growth factors in male pattern baldness surgery. Plast Reconstr Surg 118: $1458-1466$.

10. Chaudhari ND, Sharma YK, Dash K, Deshmukh P, et al. (2012) Role of platelet-rich plasma in the management of androgenetic alopecia. Int $\mathrm{J}$ Trichology 4: 291-292.

11. Greco V, Chen T, Rendl M, Schober M, Pasolli HA, et al. (2009) A two-step mechanism for stem cell activation during hair regeneration. Cell Stem Cell 4: 155-169.

12. Lopez V, Vaya A, Bautista D, Ricart JM (2013) Autologous platelet-rich plasma as a potential therapeutic tool in androgenetic alopecia. J Am Acad Dermatol 68: AB103.

13. Kang JS, Zheng Z, Choi MJ, Lee SH, Kim DY, et al. (2012) The effect of CD34+ cell-containing autologous platelet-rich plasma injection on pattern hair loss: A preliminary study. J Eur Acad Dermatol Venereol 28: 72-79.

14. Park K, Kim H, Kim BJ, Kim MN (2012) Letter: Platelet-rich plasma for treating male pattern baldness. Dermatol Surg 38: 2042-2044.

15. Greco J, Brandt R (2007) Our experience utilizing autologous platelet rich plasma in all phases of hair transplant surgery. Hair Transplant Forum Int 17: 131-132.

16. Rinaldi F, Sorbellini E, Coscera T (2011) The role of platelet rich plasma to control anagen phase: Evaluation in vitro and in vivo in hair transplant and hair treatment. Int J Trichol 3: S14-S15. 